\title{
Laboratoires - Contrôles de qualité externe
}

Jacques Weidmann

Correspondance: Jacques Weidmann FMH service tarifaire Gösgerstrasse 8 CH-4600 Olten

tarif@fmh.ch
La Commission suisse pour l'assurance qualité dans le laboratoire médical (QUALAB) existe depuis de nombreuses années. Elle a pour mandat d'ordonner des mesures de contrôle de la qualité dans les laboratoires de cabinets médicaux, ce en vertu de la Loi sur l'assurance-maladie (LAMal), de l'Ordonnance sur l'assurance-maladie (OAMAL) et de la liste des analyses publiée par le Département fédéral de l'intérieur (DFI).

Vous trouverez de nombreuses informations à ce sujet sur le site Internet de la QUALAB (www.qualab.ch). Vous pourrez notamment y consulter la liste actualisée des analyses soumises au contrôle de qualité externe. La version en vigueur pour 2006 est reproduite à la fin du présent article.

\section{Contrôle de qualité obligatoire}

Quelque 8000 membres de la FMH ont un laboratoire d'analyses médicales dans leur cabinet. Chaque praticien qui facture à la charge des assurances sociales des analyses soumises au contrôle de qualité, doit obligatoirement participer aux contrôles externes prescrits et s'affilier à l'un des trois centres de contrôle de qualité reconnus:

- CSCQ Centre Suisse de Contrôle de Qualité, Chemin du Petit-Bel-Air 2, 1225 ChêneBourg, tél. 0223055230 (français), tél. 022 3055231 (allemand), tél. 0223055232 (italien), e-mail: cscq@hcuge.ch, Internet: www.cscq.ch;

- MQ Verein für medizinische Qualitätskontrolle, c/o Institut de chimie clinique, Hôpital universitaire, 8091 Zurich, tél. 044 25534 11, e-mail: mq@unizh.ch, Internet: www. mqzh.ch;

- BSD SRK Service de transfusion sanguine de la CRS bernoise SA, Murtenstrasse 133, case postale 5512, 3001 Berne, tél. 0313842323.

\section{Sanctions en cas de non-participation aux contrôles de qualité externes}

Si la QUALAB constate que durant deux années consécutives un fournisseur de prestation, par exemple un médecin, ne s'est pas soumis aux contrôles de qualité externes obligatoires, celuici est alors inscrit dans une liste négative. Dans la $1^{\text {re }}$ liste établie pour 2004 figuraient environ 150 noms. Pour 2005, cette liste compte environ 80 noms ce qui représente un progrès notable. Les assureurs pourront sanctionner les fournisseurs de prestations dont le nom figure sur cette liste. Ils s'appuient pour cela sur l'art. 59 de la LAMal ainsi que sur les contrats QUALAB en vigueur au sujet du laboratoire.

La sanction est prononcée par un assureur. Si le fournisseur de prestation conteste cette mesure il peut alors s'adresser à la Commission paritaire de confiance qui tentera une conciliation et dont l'adresse est: Commission paritaire de confiance de la QUALAB, santésuisse, Römerstrasse 20, 4502 Soleure, tél. 03262542 23, fax 03262542 72. Si la conciliation proposée par la Commission paritaire de confiance devait échouer, ce sont alors les tribunaux cantonaux qui devraient intervenir.

\section{Liste des analyses soumises aux contrôles de qualité externe - Version valable pour l'année 2006}

Vous trouvez ci-dessous la liste des analyses soumise aux contrôles de qualité obligatoires pour l'année 2006. Cette liste ainsi que des informations complémentaires peuvent être téléchargées sur Internet: www.qualab.ch. 


\subsection{Chimie, hématologie, immunologie}

$\mathrm{M}=$ modifications par rapport à 2005: $\mathrm{N}$ = nouveau; $\mathrm{C}=$ changement de critère; $\mathrm{S}=$ supprimé; $\mathrm{Q}=$ changement de CCQ reconnu.

\begin{tabular}{|c|c|c|c|c|c|}
\hline M & no position & paramètre & $\begin{array}{l}\text { critères } \\
\text { de qualité }\end{array}$ & $\begin{array}{l}\text { critères } \\
\text { de nombre }\end{array}$ & $\begin{array}{l}\text { CCQ reconnus } \\
\text { (cf. p. 1) }\end{array}$ \\
\hline$S$ & 8000.00 & Groupes sanguins & juste & $11 / 12$ & c \\
\hline $\mathrm{N}$ & 8001.00 & $A B O$, groupe sanguin et antigène $D$ & juste & $12 / 12$ & c \\
\hline \multirow[t]{3}{*}{ C } & 8006.00 & Alanine-aminotransférase (ALAT) & $\pm 21 \%$ & $75 \%$ & $a, b$ \\
\hline & 8007.00 & Albumine, chimique & $\pm 15 \%$ & $75 \%$ & $a, b$ \\
\hline & 8017.00 & Alpha-1-foetoprotéine (AFP) & $\pm 25 \%$ & $75 \%$ & $a, b$ \\
\hline \multirow[t]{2}{*}{ C } & 8012.00 & Phosphatase alcaline & $\pm 21 \%$ & $75 \%$ & $a, b$ \\
\hline & 8036.00 & Amphétamines, ql & juste & $75 \%$ & $a, b$ \\
\hline C & 8037.00 & Amylase & $\pm 30 \%$ & $75 \%$ & $a, b$ \\
\hline \multirow[t]{6}{*}{ C } & 8058.00 & Aspartate-aminotransférase (ASAT) & $\pm 21 \%$ & $75 \%$ & $a, b$ \\
\hline & 8116.00 & Barbituriques, ql & juste & $75 \%$ & $a, b$ \\
\hline & 8119.00 & Benzodiazépines, ql & juste & $75 \%$ & $a, b$ \\
\hline & 8126.00 & Bilirubine totale & $\pm 20 \%$ & $75 \%$ & $a, b$ \\
\hline & 8129.00 & Gazométrie: $\mathrm{pH}$ & $\pm 1 \%$ & $75 \%$ & $a, b$ \\
\hline & 8129.00 & Gazométrie: $\mathrm{pO}_{2}, \mathrm{pCO}_{2}$ & $\pm 15 \%$ & $75 \%$ & $a, b$ \\
\hline \multirow[t]{3}{*}{ C } & 8137.00 & Protéine $\mathrm{C}$ réactive (CRP), qn & $\pm 21 \%$ & $75 \%$ & $a, b$ \\
\hline & 8149.00 & Calcium total & $\pm 12 \%$ & $75 \%$ & $a, b$ \\
\hline & 8152.00 & Antigène carcino-embryonnaire (CEA) & $\pm 25 \%$ & $75 \%$ & $a, b$ \\
\hline \multirow[t]{5}{*}{ C } & 8156.00 & Chlorures & $\pm 9 \%$ & $75 \%$ & $a, b$ \\
\hline & 8158.00 & Cholestérol total & $\pm 10 \%$ & $75 \%$ & $a, b$ \\
\hline & 8169.00 & Cocaïne, ql & juste & $75 \%$ & $a, b$ \\
\hline & 8174.00 & Cortisol, basal & $\pm 20 \%$ & $75 \%$ & $a, b$ \\
\hline & 8179.00 & D-dimère, ql & juste & $75 \%$ & $a, b$ \\
\hline \multirow[t]{9}{*}{ C } & 8179.01 & D-dimère, qn & $\pm 21 \%$ & $75 \%$ & $a, b$ \\
\hline & 8184.00 & Cannabis, ql & juste & $75 \%$ & $a, b$ \\
\hline & 8192.00 & Fer & $\pm 20 \%$ & $75 \%$ & $a, b$ \\
\hline & 8199.00 & $\begin{array}{l}\text { Erythrocytes alloanticorps, } \\
\text { spécification }\end{array}$ & juste & $100 \%$ & c \\
\hline & 8200.00 & $\begin{array}{l}\text { Erythrocytes alloanticorps, } \\
\text { test de recherche }\end{array}$ & juste & $100 \%$ & c \\
\hline & 8210.00 & Erythrocytes, numération & $\pm 25 \%$ & $75 \%$ & $a, b$ \\
\hline & 8225.00 & Ferritine & $\pm 25 \%$ & $75 \%$ & $a, b$ \\
\hline & 8229.00 & Fibrinogène, d'après Clauss & $\pm 15 \%$ & $75 \%$ & $a, b$ \\
\hline & 8239.00 & Folates & $\pm 20 \%$ & $75 \%$ & $a, b$ \\
\hline \multirow[t]{3}{*}{ C } & 8249.00 & $\begin{array}{l}\text { Gamma-glutamyltranspeptidase } \\
(\gamma-\mathrm{GT})\end{array}$ & $\pm 21 \%$ & $75 \%$ & $a, b$ \\
\hline & 8259.00 & Glucose & $\pm 10 \%$ & $75 \%$ & $a, b$ \\
\hline & 8265.00 & Hémoglobine glyquée (HbA1c) & $\pm 15 \%$ & $75 \%$ & $a, b$ \\
\hline C & 8273.00 & Hématocrite & $\pm 9 \%$ & $75 \%$ & $a, b$ \\
\hline \multirow[t]{9}{*}{ C } & 8275.00 & Hémoglobine & $\pm 9 \%$ & $75 \%$ & $a, b$ \\
\hline & 8284.00 & Urée & $\pm 20 \%$ & $75 \%$ & $a, b$ \\
\hline & 8286.00 & $\begin{array}{l}\text { Human Choriongonadotropin } \\
\text { (HCG), qn }\end{array}$ & $\pm 25 \%$ & $75 \%$ & $a, b$ \\
\hline & 8288.00 & $\begin{array}{l}\text { Cholestérol HDL sans précipitation } \\
\text { séparée }\end{array}$ & $\pm 30 \%$ & $75 \%$ & $a, b$ \\
\hline & 8313.00 & Immunoglobuline IgA (sérum) & $\pm 25 \%$ & $75 \%$ & $a, b$ \\
\hline & 8317.00 & Immunglobuline IgE total, qn & $\pm 20 \%$ & $75 \%$ & $a, b$ \\
\hline & 8317.01 & Immunoglobuline IgE multispécifique & juste & $75 \%$ & $a, b$ \\
\hline & 8317.02 & IgE spécifique - arachides & \pm 1 classe & $75 \%$ & $a, b$ \\
\hline & 8317.02 & IgE spécifique - bouleau & \pm 1 classe & $75 \%$ & $a, b$ \\
\hline
\end{tabular}




\subsection{Chimie, hématologie, immunologie (suite)}

$\mathrm{M}=$ modifications par rapport à 2005: $\mathrm{N}$ = nouveau; $\mathrm{C}=$ changement de critère; $\mathrm{S}=$ supprimé; $\mathrm{Q}=$ changement de CCQ reconnu.

\begin{tabular}{|c|c|c|c|c|c|}
\hline & 8317.02 & IgE spécifique - épithélium du chat & \pm 1 classe & $75 \%$ & $a, b$ \\
\hline & 8317.03 & IgE spécifique - arachides qn & $\pm 20 \%$ & $75 \%$ & $a, b$ \\
\hline & 8317.03 & IgE spécifique - bouleau qn & $\pm 20 \%$ & $75 \%$ & $a, b$ \\
\hline & 8317.03 & IgE spécifique - épithélium du chat qn & $\pm 20 \%$ & $75 \%$ & $a, b$ \\
\hline & 8318.00 & Immunoglobuline IgG (sérum) & $\pm 25 \%$ & $75 \%$ & $a, b$ \\
\hline & 8321.00 & Immunoglobuline IgM (sérum) & $\pm 25 \%$ & $75 \%$ & $a, b$ \\
\hline$C$ & 8343.00 & Potassium & $\pm 9 \%$ & $75 \%$ & $a, b$ \\
\hline \multirow[t]{2}{*}{ C } & 8384.00 & Créatine-kinase (CK), total & $\pm 30 \%$ & $75 \%$ & $a, b$ \\
\hline & 8387.00 & Créatinine & $\pm 20 \%$ & $75 \%$ & $a, b$ \\
\hline \multirow[t]{6}{*}{ C } & 8394.00 & Lactate-déshydrogénase (LDH) & $\pm 21 \%$ & $75 \%$ & $a, b$ \\
\hline & 8403.00 & Leucocytes, répartition & * & $75 \%$ & $a, b$ \\
\hline & 8406.00 & Leucocytes, numération & $\pm 25 \%$ & $75 \%$ & $a, b$ \\
\hline & 8428.00 & Magnésium & $\pm 20 \%$ & $75 \%$ & $a, b$ \\
\hline & 8433.00 & Méthadone, ql & juste & $75 \%$ & $a, b$ \\
\hline & 8437.00 & Myoglobine & $\pm 30 \%$ & $75 \%$ & $a, b$ \\
\hline \multirow[t]{12}{*}{ C } & 8438.00 & Sodium & $\pm 9 \%$ & $75 \%$ & $a, b$ \\
\hline & 8444.00 & Opiacés, ql & juste & $75 \%$ & $a, b$ \\
\hline & 8450.00 & Amylase pancréatique & $\pm 25 \%$ & $75 \%$ & $a, b$ \\
\hline & 8455.10 & $\begin{array}{l}\text { Temps de thromboplastine partielle } \\
\text { activée (aPTT) }\end{array}$ & $\pm 25 \%$ & $75 \%$ & $a, b$ \\
\hline & 8462.00 & Phosphate & $\pm 15 \%$ & $75 \%$ & $a, b$ \\
\hline & 8485.00 & Prostate, antigène spécifique (PSA) & $\pm 25 \%$ & $75 \%$ & $a, b$ \\
\hline & 8485.01 & $\begin{array}{l}\text { Prostate, antigène spécifique (PSA), } \\
\text { libre }\end{array}$ & $\pm 25 \%$ & $75 \%$ & $a, b$ \\
\hline & 8492.00 & Protéines totales & $\pm 15 \%$ & $75 \%$ & $a, b$ \\
\hline & 8548.00 & Quick & $\pm 15 \%$ & $75 \%$ & $a, b$ \\
\hline & 8560.00 & Thrombocytes, numération & $\pm 25 \%$ & $75 \%$ & $a, b$ \\
\hline & 8562.00 & Thyroxine libre (FT4) & $\pm 20 \%$ & $75 \%$ & $a, b$ \\
\hline & 8572.00 & Triglycérides & $\pm 20 \%$ & $75 \%$ & $a, b$ \\
\hline \multirow[t]{3}{*}{$C$} & 8574.10 & Troponine (T ou I), par méthode ELISA & $\pm 24 \%$ & $75 \%$ & $a, b$ \\
\hline & 8576.00 & Thyréotropine (TSH), basale & $\pm 20 \%$ & $75 \%$ & $a, b$ \\
\hline & 8577.00 & Thyréotropine (TSH), stimulée & $\pm 20 \%$ & $75 \%$ & $a, b$ \\
\hline \multirow[t]{3}{*}{$C$} & 8578.00 & Urate & $\pm 15 \%$ & $75 \%$ & $a, b$ \\
\hline & 8587.00 & $\begin{array}{l}\text { Test de compatibilité, test ind. } \\
\text { antiglob. humainesc }\end{array}$ & juste & $100 \%$ & c \\
\hline & 8590.00 & Vitamine B12 & $\pm 20 \%$ & $75 \%$ & $a, b$ \\
\hline
\end{tabular}

Les paramètres ci-dessus sont aussi soumis au contrôle de qualité externe obligatoire, lorsqu'ils sont facturés dans le cadre d'un bloc d'analyses sous un autre numéro, ou si le résultat est obtenu suite à un test clinique. 


\subsection{Microbiologie}

\subsubsection{Virologie}

\begin{tabular}{lllll}
9108.40 & Hépatite $B$, virus, HBs, recherche des antigènes, ql & pas de faux nég. & $100 \%$ & $a, c$ \\
\hline 9109.01 & Hépatite , virus, anticorps lg ou lgG, ql & pas de faux nég. & $100 \%$ & $a, c$ \\
\hline 9116.01 & HIV $1+2$, dépistage des anticorps (EIA), ql & pas de faux nég. & $100 \%$ & $a, c$ \\
\hline 9116.40 & HIV $1+2$, dépistage des anticorps (test rapide), ql & pas de faux nég. & $100 \%$ & $a, b$
\end{tabular}

\subsubsection{Bactériologie}

L'ensemble des analyses de bactériologie du chapitre 3.2.2.

de la liste fédérale des analyses

Analyses des soins de base (chapitre 5.1.3 de la liste des analyses)

$\begin{array}{lllll}9309.00 & \text { Urine slide } & \text { juste } & 75 \% & a, b \\ 9559.72 & \begin{array}{l}\text { Streptococcus bêta-hémolytique du groupe A } \\ \text { (par méthode rapide) }\end{array} & \text { juste } & 75 \% & \text { a, b }\end{array}$

\section{Remarque}

Le contrôle de qualité effectif pour les analyses du chapitre 1.2.2 LA est réalisé sur mandat du CSCQ et de MQ par les centres de contrôle suivants: NEQAS (pour le CSCQ) et IMM (pour MQ). CSCQ et MQ assurent le contact avec les laboratoires. Une adhésion directe à NEQAS ou IMM n'est pas reconnue dans le cadre du contrôle de qualité obligatoire.

\subsection{Génétique}

Pour des raisons techniques, un seul contrôle externe est organisé pour ces analyses. Par contre, plusieurs échantillons doivent être examinés lors de ce contrôle.

Tous les laboratoires qui effectuent les analyses ci-dessous doivent envoyer chaque année à la QUALAB une copie de l'évaluation resp. du certificat, et ceci dès réception du document, mais au plus tard pour la fin de l'année.

\section{Evaluation du contrôle de qualité externe obligatoire}

Lors de l'évaluation des résultats du contrôle de qualité externe, il y a lieu de tenir compte des points suivants:

\begin{tabular}{|c|c|c|c|c|c|}
\hline M & no position & paramètre / maladie & $\begin{array}{l}\text { critères } \\
\text { de qualité }\end{array}$ & $\begin{array}{l}\text { critères } \\
\text { de nombre }\end{array}$ & $\begin{array}{l}\text { CCQ reconnus } \\
\text { (cf. p. 1) }\end{array}$ \\
\hline & 8801.00 & Caryotype constitutionnel & juste & $1 / 1$ & $e, f$ \\
\hline & 8802.00 & $\begin{array}{l}\text { Analyse chromosomique, hémopathies } \\
\text { malignes }\end{array}$ & juste & $1 / 1$ & $f$ \\
\hline & 8805.00 & FISH métaphase & juste & $1 / 1$ & $e, f$ \\
\hline & 8806.00 & FISH interphase & juste & $1 / 1$ & $e, f$ \\
\hline & 8810.01 & Angelman / Prader Willi & juste & $1 / 1$ & d, e \\
\hline & 8810.05 & Microdélétion du chromosome $Y$ & juste & $1 / 1$ & $d$ \\
\hline \multirow[t]{2}{*}{ Q } & 8810.07 & Mucoviscidose & juste & $1 / 1$ & $g$ \\
\hline & 8810.08 & Syndrome de I'X-fragile & juste & $1 / 1$ & $d, e$ \\
\hline \multirow[t]{6}{*}{ Q } & 8810.11 & Hémochromatose & juste & $1 / 1$ & $a, b, d, e$ \\
\hline & 8810.13 & Maladies d'hémostase & juste & $1 / 1$ & $a, b$ \\
\hline & 8810.19 & $\begin{array}{l}\text { Cancer du sein / Carcinomes du colon } \\
\text { héréditaires / Rétinoblastome }\end{array}$ & juste & $1 / 1$ & d, e \\
\hline & 8810.23 & Dystrophie musculaire de Duchenne & juste & $1 / 1$ & d, e \\
\hline & 8810.27 & $\begin{array}{l}\text { Dystrophie myotonique / Ataxie de } \\
\text { Friedreich / Huntington Disease / } \\
\text { Ataxies spinocérébelleuse }\end{array}$ & juste & $1 / 1$ & e \\
\hline & 8810.32 & Charcot-Marie-Tooth & juste & $1 / 1$ & d, e \\
\hline
\end{tabular}


- En médecine de laboratoire, les méthodes et les systèmes analytiques utilisés sont très diversifiés. Ceci peut conduire à une hétérogénéité des résultats du contrôle de qualité. Il n'est donc pas toujours possible de fixer des critères généraux ou des valeurs cibles globales.

- La qualité des résultats du contrôle de qualité est influencée par la qualité du matériel de contrôle. Pour des raisons techniques il n'est pas toujours possible de mettre à disposition un matériel de contrôle optimal pour chaque système analytique. Les centres de contrôle de qualité doivent tenir compte de cet élément lors de l'évaluation des résultats.

- Pour les raisons ci-dessus, plusieurs cas spéciaux doivent être pris en compte lors de l'évaluation de la qualité des résultats des contrôles de qualité. Il n'est pas toujours possible de les connaître à l'avance. Aussi et dans le but de permettre des contrôles rapides et administrativement gérables, une certaine liberté d'appréciation est accordée à titre d'essai aux centres de contrôle de qualité. Cette liberté d'appréciation est également valable pour les paramètres nouvellement introduits. Les centres de contrôle de qualité rapportent à la fin de l'année les cas spéciaux, dans lesquels cette liberté d'appréciation a été utilisée.

\subsection{Paramètres avec ou sans contrôle de qualité externe obligatoire}

\subsubsection{Paramètres avec contrôle de qualité} externe obligatoire (selon point 1 du présent document)

La participation annuelle à au moins quatre contrôles (rendu de quatre résultats par analyse) est obligatoire. Lorsque la QUALAB n'exige dans des cas exceptionnels ou pour certaines analyses spéciales - que deux contrôles, la participation à ces deux contrôles est nécessaire.

La qualité des analyses est évaluée selon les critères fixés par la QUALAB.

\subsubsection{Paramètres sans contrôle de qualité externe obligatoire (ne figurant pas sur la liste du point 1)}

Il n'existe pas de directives (critères de qualité) établies par la QUALAB.

\subsection{Critères d'évaluation / certificat}

2.2.1 Critères de qualité, critères de nombre La qualité analytique de chaque paramètre est évaluée au courant de l'année. En règle générale, les conditions sont remplies, lorsque $75 \%$ des ré- sultats des contrôles annuels de chaque paramètre correspondent aux critères de la QUALAB. Certains paramètres peuvent avoir d'autres exigences (voir colonne «critères de nombre» du tableau au point 1$)$.

\subsubsection{Certificat}

Le certificat comprend une liste des paramètres déclarés par le laboratoire et contrôlés (avec numéros de position de la LA), le nombre total de contrôles par paramètre et le nombre de résultats conformes.

Le certificat doit porter l'identification unique, le nom et l'adresse du laboratoire, son numéro RCC (anciennement numéro de concordat), la période couverte par le contrôle, l'identification du CCQ qui l'a délivré ainsi que le nom et le titre de la personne qui valide le certificat.

\subsubsection{Nouvelles inscriptions / changement d'inscriptions}

Si un laboratoire introduit une nouvelle analyse ou commence le contrôle de qualité en cours d'année, le certificat mentionne le total pro rata temporis des résultats à rendre.

Ces participants ne sont pas dispensés d'analyser les résultats non conformes et de consigner les mesures prises.

\subsection{Obligations des laboratoires}

\subsubsection{Participation aux contrôles de qualité externes}

Si un laboratoire réalise des analyses mentionnées au tableau du point 1 , il doit s'inscrire au nombre de contrôles de qualité externes prescrit et ce auprès d'un centre de contrôle de qualité reconnu par la QUALAB. Pour les paramètres de la liste des soins de base (cf. liste fédérale des analyses), seuls des centres de contrôle de qualité suisses sont reconnus. Pour tous les autres paramètres, des centres de contrôle de qualité étrangers peuvent également être reconnus sur demande des sociétés scientifiques.

\subsubsection{Procédure lorsque les critères d'évaluation ne sont pas remplis}

Le laboratoire est tenu de rechercher les causes des résultats non conformes et d'en conserver la documentation (5 ans au minimum).

Si les critères de qualité et les critères de nombre pour un paramètre ne sont pas remplis, le laboratoire doit prendre contact avec le centre de contrôle de qualité pour tenter d'identifier la source du problème. 